\title{
La confluencia entre raperos chilenos y sellos discográficos multinacionales en la década de 1990:
}

\author{
Una operación con implicancias para el desarrollo del \\ hip-hop en Chile
}

\begin{abstract}
NELSON RODRÍGUEZ
Resumen: El artículo estudia la confluencia entre raperos chilenos y sellos discográficos multinacionales (majors) en la década de 1990. Este período se distingue por una serie de cambios en el despliegue del hip-hop en Chile. La injerencia de las majors fue importante porque consolidó una red de soporte integrada también por otros agentes, entre cuyos resultados estuvo que el rap/hip-hop alcanzó cierta ratificación social, artística, cultural y de productividad. Por otro lado, esta operación es comúnmente desatendida a nivel académico. Se infiere que el resultado referido no armoniza, de alguna forma, con la habitual representación contrahegemónica del hip-hop, lo cual ha significado un vacío bibliográfico. En última instancia, este artículo intenta demostrar que la vinculación con la industria cultural/mercado no se traduce necesariamente en la pérdida de la función social de la música y que, además, existirian formas alternativas de ejercer una resistencia cultural no ceñidas a efectuar irremediablemente una crítica social en letras de canciones, o a la toma de una posición política explícita, por parte de los músicos populares.
\end{abstract}

Palabras claves: Rap/hip-hop chileno, Sellos discográficos multinacionales, Industria cultural, Década de 1990.

\section{The confluence between Chilean rappers and multinational record labels during the decade of 1990: an operation with implications for the development of hip-hop in Chile}

\begin{abstract}
The article studies the confluence between Chilean rappers and multinational record labels (majors) in the 1990s. This period is distinguished by various changes in the deployment of hip-hop in Chile. The interference of the majors was important because it consolidated a support network also made up by other agents, which result was that, to some extent, the aforementioned musical culture achieved a social, artistic, cultural and productivity

\footnotetext{
* Nelson Leandro Rodríguez Vega es estudiante del programa Doctorado en Artes de la Pontificia Universidad Católica de Chile. Becario de la Agencia Nacional de Investigación y Desarrollo (ANID). Magíster en Artes mención Musicología por la Universidad de Chile. Profesor de Música y Licenciado en Educación por la Universidad de Concepción de Chile. Investigador especializado en el estudio del hip-hop chileno. E-mail: ne.rodriguez@uc.cl
} 
ratification. On the other hand, this is a commonly disregarded operation on academic level. It's inferred that the aforementioned encounter in some way does not harmonize with the usual counter-hegemonic representation of hip-hop, which has meant a bibliographic gap. At the last, the article tries to show that a link with the cultural industry/market does not necessarily translate into a loss of a social function of music, and also would be exist alternative ways of applying a cultural resistance not limited to making social criticism in song lyrics, or taking an explicit political position on the part of popular musicians.

Keywords: Chilean rap/hip-hop, Multinational record labels, Cultural industry, Decade of 1990

\section{Introducción}

$\mathrm{E}$ ste artículo profundiza en la confluencia entre raperos chilenos y sellos discográficos multinacionales (majors) en la década de 1990. En dicho momento se comenzó a avizorar una transformación del hip-hop en Chile, notable en ámbitos como el despliegue de la cultura, la circulación de la música, la ampliación de la comunidad, el nivel sonoro, entre otros. Así las cosas, y a partir de un panorama de masividad del hip-hop en Chile, la escena nacional lograría posicionarse con relevancia en América Latina. Desde este prisma, señalo que la confluencia entre músicos y majors, junto con la temporalidad en cuestión, representan un valioso lugar para pensar y (re) significar al hip-hop chileno.

Durante los años previos a la década de 1990, el hip-hop en Chile contaba con una exigua visibilidad en la sociedad y un desarrollo parsimonioso. Subyace a estas condiciones el reducido número de grabaciones por parte de bandas nacionales de rap, dado que los escasos álbumes habían surgido desde la autogestión o, en algunos casos, a partir de sellos independientes (minors), pero con las correspondientes limitantes para la producción, grabación y difusión del producto musical. De tal modo, el pequeño universo de bandas solía ser escuchado entre un público reducido y acotado. Con la injerencia de las majors se acabaría de consolidar una red de soporte, integrada también por otros agentes, obteniendo como resultado que el hip-hop chileno alcanzara, en cierta medida, una ratificación social, artística, cultural y de productividad. 
Con todo, es pertinente consignar la presencia de un aura de cierta negatividad que suele recaer sobre la operación consignada, e incluso sobre el período en estudio. Así pues, algunos hiphoperos chilenos, principalmente pertenecientes a la generación pionera de los años ochenta, suelen manifestar desdén ante lo que habría sido una supuesta banalización de la cultura, dado el alto grado de comercialización y exposición en los medios de comunicación. De alguna manera este sentir es compartido por algunos investigadores académicos, porque asocian la industria discográfica multinacional, o la industria cultural en general, con una sospechosa mercantilización del arte (POCH, 2011; ARAVENA, 2011) ${ }^{1}$.

El rap de países periféricos recurrentemente potencia discursos de denuncia y al mismo tiempo toma distancia de atributos comerciales (ÁVILA; LIBERATO; CONTE, 2017, p. 202). Dicha tesis es reafirmada por numerosas investigaciones especializadas en hip-hop chileno, ya que se ha preferido resaltar la faceta política y de crítica social de raperos locales (MORAGA; SOLORZANO, 2005, QUITZOW, 2005, TIJOUX; FACUSE; URRUTIA, 2012, MARTIN-CABRERA, 2016).

En última instancia, el presente artículo reflexiona superficialmente acerca de la función social de la música. Se conjetura que una vinculación con la industria cultural no necesariamente se traduce en una pérdida irremediable de lo social. Para nuestro caso, por ejemplo, la vitrina que proporcionaron los sellos multinacionales facilitó el conocimiento e identificación de más sujetos con el hip-hop, así como le permitió a algunos raperos difundir mensajes entre audiencias más amplias. Siguiendo esta línea, lo político no resultaría ser el único acceso a una función social de la música, ya que algunos investigadores latinoamericanos han demostrado formas alternativas de ejercer una resistencia cultural, más allá de un prisma rígido de entender la hegemonía y contrahegemonía. Por tanto, atender este tipo de relaciones desde una mirada no prejuiciosa, pero igualmente crítica, podría devenir en un enriquecimiento

\footnotetext{
1 Poch (2011, p. 127) articula su crítica hacia los sellos discográficos multinacionales desde los ejemplos de M-16 y Tiro de Gracia. Indica que EMI ofreció un contrato a la primera banda, pero éste fue desechado para no transar el contenido político de las letras sólo a cambio de réditos económicos. Tal descarte llevaría a contratar a Tiro de Gracia, banda que precisamente dio inicio al auge comercial y mediático del hip-hop en Chile durante la década de 1990. Por su parte Aravena (2011, p. 205) sostiene que uno de los cánones prestablecidos del hip-hop chileno es no relacionarse con el mercado.
} 
epistemológico de la investigación de prácticas musicales cercanas a una idea de lo popular.

\section{El hip-hop chileno en años previos al boom}

El hip-hop suele ser definido como una cultura integrada por el break dance, el rap, la figura del DJ y el fenómeno (o la expresión visual) del grafiti ${ }^{2}$. Su correspondiente origen es localizado en el despertar de la década de 1970 en el barrio neoyorkino del Bronx, Estados Unidos. En su articulación habría desempeñado un rol preponderante el contexto social, pues es habitual la referencia a diferentes problemáticas sociales que afectaban a una población integrada mayoritariamente por afroamericanos y latinos (CHANG, 2015). Sería por medio del hip-hop que muchos jóvenes tuvieron la oportunidad de escapar simbólicamente a su entorno social (PACINI, 2009, p. 56).

Por otro lado, fue a finales de los años setenta y comienzos del siguiente decenio que el hip-hop se convirtió en objeto de interés de la industria cultural norteamericana. Así, algunos sellos discográficos, productoras audiovisuales, estudios cinematográficos, galerías de arte, entre otros espacios asociados a la cultura y a las artes, articularon su emergencia dando paso, así, a su exportación a otras latitudes (CHANG, 2015, p. 175).

Particularmente en Chile, las primeras expresiones de hip-hop tuvieron lugar en la televisión local (MENESES, 2014, p. 10). El break dance habría sido el primer elemento presentado, aunque preliminarmente los jóvenes nacionales desconocían la pertenencia de dicha danza a la cultura del hip-hop (POCH, 2011, p. 70). Una aproximación más acabada se concretaría por la mediación del cine y también por la migración. En relación a este último agente, se trató en específico de hijos de exiliados de la dictadura de Augusto Pinochet, quienes a su arribo/retorno trajeron vasta

\footnotetext{
2 Entre las diferentes manifestaciones artísticas que integran la cultura del hip-hop, el rap (género musical) ha adquirido una mayor relevancia justificado por las cuantiosas ganancias que genera a la industria discográfica. Así se han relegado las otras expresiones a un plano secundario (KATZ, 2012, p. 70). Actualmente se suele utilizar el término hip-hop para referirse al género musical. En este escrito se prosigue dicha tendencia.
} 
información proveniente de territorios en que el hip-hop se encontraba más afianzado. Estos saberes fueron divulgados en los incipientes puntos de reunión, ubicados preferentemente en la capital, Santiago (RODRÍGUEZ, 2020a, p. 94).

A partir de 1988, el hip-hop experimentó en Chile dos circunstancias que dispusieron su trayectoria en los años venideros. Una, fue el declive de popularidad que experimentó el break dance ante su desestimación como novedad mundial. Este hecho se manifestó tanto en una desatención por parte de la industria cultural chilena, como por el arribo de otras categorías musicales foráneas que, de igual manera, llegaron a satisfacer el gusto musical y articular la experiencia de juventud. La segunda circunstancia fue la mayor estimación que adquirió el rap (género musical) entre la comunidad del hip-hop, hecho que se inscribió en una tendencia globalizada (CHANG, 2015, p. 173). Fue en este contexto que se suscitaron las inaugurales aspiraciones del rap chileno, las cuales suelen ser recordadas por sus protagonistas como "precarias" y de "escasa visibilidad" social.

Como posible justificativa de este tipo de apreciación cuentan dos elementos, al menos. El primero sería la falta de acceso a insumos para generar música como fondo sonoro a la línea vocal de las canciones (tornamesas de DJ, samplers, máquinas secuenciadoras de ritmo) porque, simplemente, estos aún no se comercializaban en el territorio. Aun cuando se generaron alternativas para resolver tal dilema - grabación de fragmentos sonoros resignificados a través de radios de doble-casetera-, la calidad de estas inusuales bases no era del todo óptima (RODRÍGUEZ, 2019, p. 69).

Experimentar a la antigua, como el tema de hacer un breakbeat (base musical) con cintas de casetes. Buscabas el espacio y lo volvías a repetir. Tenías que construir toda una cinta, cortándola, editándola, editando un casete para darle una vuelta. No había nada equipos. Si había equipos nosotros nunca lo habíamos tenido porque teníamos la necesidad de comer también en el barrio. No era fácil tener una tornamesa. Al principio, de hecho, cuando tuvimos el primer DJ, el maestro "Carquín", él tenía una tornamesa que tenía una salida y esa salida tenía un parlante, y nosotros poníamos el micrófono al parlante y así el hombre podía scratchear. Se amplificaba el parlante, no era salir con el mezclador, no existía para nosotros esos equipos. A finales de los noventa se pulió la esfera o "la piedra", sacaron más brillo, había más recursos. Claro, y ahí se puso la "guita" (dinero) y estaban los sellos. En ese entonces había sellos que perfectamente te podían pagar el estudio, y el estudio valía tantas "lucas" (pesos chilenos). Tú podías enganchar con hacer algo un poquito más 
profesional (Comunicación personal con Daniel Fernández, 20 de noviembre de 2017).

Un segundo elemento estaba relacionado con la divulgación y exhibición de la música. Específicamente radios masivas y con alcance nacional no mostraron un mayor interés por el incipiente rap chileno. Algunos raperos de esta primera generación apuntan a una directa censura, bien sea porque las letras abordaban problemáticas sociales o por la presencia de lenguaje soez (uso de malas palabras). Otros aluden que la baja calidad del producto musical atentó contra su circulación radial extendida. Así las cosas, las reducidas ocasiones en que una radio importante reprodujo alguna grabación de este tipo, se optó por irradiar exponentes internacionales o de alguna canción hispanoamericana que apelaba a la sonoridad del género, aunque la mayoría de las veces en clave de parodia (RODRÍGUEZ, 2019, p. $65)$.

En lo que respecta a presentaciones, las primeras bandas nacionales de rap contaron con acotadas instancias para socializar su música en vivo. Los pocos espacios que generaban oportunidades de "tocar" se caracterizaban por una propensión política o solidaria. Se trataba en muchos casos de actos de repudio a la dictadura o de apoyo a presos políticos durante los primeros años del retorno a la democracia (MENESES, 2014).

Por otra parte, se suele indicar que el rap chileno ingresó a la industria discográfica recién a partir de la segunda mitad de la década de 1990. Sin embargo, en años precedentes algunas bandas sí tuvieron la oportunidad de grabar con un sello discográfico. Se trató, en concreto, de minors que observaron en esta "música extranjera" una forma de seguir afianzando una identidad de propensión política. La banda pionera fue Panteras Negras que grabó su primer álbum, Lejos del centro (1991), con el sello Liberación, vinculado al Partido Comunista de Chile. Posteriormente Los Marginales y Estrellas de la Calle grabaron sus debuts discográficos con Prodisc y Pollo Records, respectivamente. Pero quizás la asociación más significativa fue la que formó el sello Alerce con Panteras Negras y La Pozze Latina. Este sello había surgido en plena dictadura militar para apoyar al canto popular, pero a comienzos de la década 
de 1990 se abrió a la posibilidad de impulsar renovadas expresiones musicales juveniles (CANALES, 2017).

No obstante, los sellos independientes tenían variadas limitantes como, por ejemplo, producir cantidades reducidas de casetes, comercializarlos en espacios acotados, y no poder promocionar a las bandas en medios de comunicación de vasto alcance. Estas condiciones tendían a agudizarse con aquellos grupos que grabaron demos desde la autogestión.

Como veremos, el hip-hop chileno experimentó una transformación durante la última parte de los años noventa. Una de las aristas que impulsó este cambio fue el rol que ejercieron las majors. En el renovado panorama, la sociedad chilena en toda su amplitud accedió a una cultura musical que superficialmente se dio a conocer en el decenio anterior, pero que en esta oportunidad tenía una aplicación a gran escala productiva. Como resultado, se sentarían las bases para un despliegue más fluido, contribuyendo así a visibilizar las propias representaciones, identidades y discursividades de los exponentes locales de esta cultura musical en territorio chileno.

\section{La confluencia entre raperos chilenos y sellos discográficos multinacionales}

Durante los últimos años de la década de 1990 el hip-hop chileno alcanzó niveles de exposición mediática y desarrollo -en producción, circulación y sonoridad - inéditos hasta ese momento. Diversos factores confluyeron para dar cauce a esta transformación, entre ellos, el retorno a la democracia, la recuperación económica, el mejor acceso a la información, la entrada al país de renovadas tecnologías para producir y grabar música (autogestión) y el rol que desempeñaron las minors. Es necesario también mencionar la expansión de la industria discográfica multinacional en Chile.

La influencia de majors no resulta ser un hecho inédito. Como muchas investigaciones han apuntado, este tipo de agentes de la industria cultural ha estado presente durante toda la segunda mitad del siglo XX, y ha resultado fundamental para 
impulsar y visibilizar a diferentes artistas y/o movimientos musicales. Sin ir más lejos, durante los años ochenta se gestó un acotado apogeo del pop que tuvo el soporte de sellos importantes como EMI, BMG, RCA, CBS y Musicavisión (PONCE, 2019, p. 32). Aun cuando la estancia de estos sellos no se extendió demasiado, sí resultó ser un precedente importante para lo que vendría algunos años después. Durante el posterior decenio, la música chilena, en general, experimentó un auge, gracias a la reinstalación de las sucursales locales de las mencionadas majors, junto con la apertura de otras.

En efecto, la década de 1990 en Chile se reconoce por su prosperidad en materia musical, ante el impulso comercial y mediático que efectuaron sellos multinacionales. Así las cosas, diversos artistas provenientes de diferentes categorías musicales accedieron a recursos económicos para grabación y difusión (EVANS; SADE, 2008). De tal manera, Chile consiguió ponerse a la par de mercados musicales regionales históricamente más relevantes (ALDUNATE, 2019, p. 117).

Los más beneficiados por esta apertura fueron aquellos proyectos vinculados a una escucha juvenil - bandas de pop o rock en sus diferentes variantes de estilo - . Cabe señalar que el sello pionero en este ámbito fue EMI con su proyecto Rock Nacional, iniciativa que fue rápidamente replicada por BMG, Polygram, SONY, Warner y Universal Music (EVANS; SADE, 2008, p. 15).

El rap en concreto fue uno de los últimos géneros "juveniles" en formar parte de la tendencia. Así y todo, prontamente el hip-hop logró generar un fuerte impacto, principalmente por las notables ventas de discos. Esta excelente posición sería ratificada además por una alta exposición en medios de comunicación y la proliferación de espacios para presentarse en vivo. Por ende, el renovado escenario difería ostensiblemente de los años anteriores cuando el rap local no contaba con demasiada visibilidad, ni tampoco su comunidad se caracterizaba por ser una de las más numerosas, en el creciente acervo de culturas musicales juveniles.

A eso yo me refiero con el asunto de los sellos. Nosotros partimos cuando no había nada, ni siquiera los sellos estaban algo interesados en saber lo que estaba pasando con la música urbana. Cuando nosotros íbamos a tocar teníamos que compartir escenario con bandas punkis, death metal, thrash. Era la música de los jóvenes que había. Los pocos raperos éramos nosotros. Cuando nosotros tocábamos, los Panteras Negras iban para abajo y eran nuestro grupo, y después tocaban los Panteras Negras y nosotros bajábamos 
y éramos el público (Comunicación personal con Jimmy Fernández, 27 de septiembre de 2017).

En esta confluencia entre rap chileno y majors, Tiro de Gracia fue la banda precursora. A mediados de 1997 lanzó al mercado Ser Humano (1997), por medio de EMI Music, y en su primer año comercializó alrededor de sesenta mil copias ${ }^{3}$. Esto no sólo resultaba una novedad para el rap sino también para la música chilena en general, dado el acotado tamaño de la industria local. El impacto generado por Tiro de Gracia también estuvo representado por su constante aparición en shows radiales y televisivos, sobre todo aquellos consagrados a un público juvenil.

Con esta banda en particular se visualizaron transformaciones en las estrategias de difusión de la música, las cuales serían imitadas por otros grupos. Uno de estos cambios fue la grabación de videoclips que circularon por importantes espacios musicales de la televisión abierta y por cable. Así sucedía, por ejemplo, con MTV, que para ese momento representaba un espacio fundamental para insertar a los músicos chilenos en un contexto internacional (ALDUNATE, 2019, p. 117).

A partir de la estrategia señalada, Tiro de Gracia pudo alcanzar el éxito en otros países de la región y en contextos más amplios. La banda obtuvo variadas menciones en medios escritos especializados y de gran resonancia en el mundo de la música, como la revista norteamericana Billboard. Incluso, estas loas trascendieron una esfera netamente musical, en virtud de que los integrantes de Tiro de Gracia fueron elegidos como imagen de campaña de la derogación de la ley de detención por sospecha, impulsada por el gobierno de turno en $1998^{4}$.

El integrante de Tiro de Gracia, Fabián Sánchez - alias “Lengua Dura” - , se refería en su momento a las transformaciones visualizadas en la escena del hip-hop chileno, ante la grabación a través de majors.

Y creo que todos los que están ahora es por lo que pasó con "Tiro", antes ibas a una tocada y había 20 grupos máximo, ahora hay como 40 por tocata, es cosa de ver los afiches, todos se pelean por rapear (Entrevista en revista Kultura Hip Hop, vol. 4, p. 24, 2000).

\footnotetext{
${ }^{3}$ Fuente: disponible en https:/ / www.musicapopular.cl/grupo/tiro-de-gracia/

${ }^{4}$ Fuente: disponible en https:/ / www.latercera.com/culto/2019/09/10/tiro-de-gracia-frei/
} 
La propuesta de Tiro de Gracia resultaba también atractiva por el tipo de producto musical que ofertaba. Al sobresaliente nivel de grabación se le sumaban aspectos sonoros poco explorados hasta el momento, y que asimismo se convirtieron en modelo a seguir. Entre estos destacan una excelente aptitud vocal de los cantantes - o mejoradas desde la producción - , musicalidad ecléctica a partir de la fusión con otros géneros, o beats que se complementaban con la ejecución de instrumentos musicales convencionales. Inclusive muchas canciones de Ser Humano y de los otros dos discos que la banda grabó con EMI -Decisión (1998) y Retorno de Misericordia (2001) - contaron con la participación de músicos consagrados provenientes de otros ámbitos de la música popular chilena. Los casos más emblemáticos fueron los cantantes Solo di Medina y Ema Pinto, quienes además colaboraron con otras bandas de rap del período. Así, las expresiones locales tomaron distancia de aspectos estilísticos tradicionales del rap -- por ejemplo, el uso de un canto que no formula melodías ni tonalidades (SEGRETO, 2017, p. 17) - y los cambiaron por otros elementos. Esto sería muy bien recibido por audiencias que no necesariamente pregonaban una identificación estricta con la cultura del hip-hop.

Evidentemente se trataba de un intento de depurar el género para que alcanzara un público más extenso. Dicho con otras palabras, se hizo del rap una música que pudiera ser fácilmente consumible y a la vez comercializable. Esto quedaría ratificado con la preponderancia que adquirió el estribillo cantado en la estructura de las canciones, lo que indujo a que el género se tornara más factible de disfrutar, dado que los estribillos suelen ser las secciones de mejor retención auditiva. Justamente, en una crítica musical al álbum Ser Humano, el musicólogo chileno Juan Pablo González relevaba la transformación positiva que estaba adquiriendo el rap local desde un plano sonoro.

Junto con ingeniárselas para meter un clavo de cuatro pulgadas dentro de la caja del disco, los Tiro de Gracia han logrado producir un sonido limpio, bien mezclado y con planos sonoros claramente diferenciados, donde se aprecia su rítmico contrapunto de timbres y ataques. Debajo del incesante discurso de la voz, aparecen teclados con armonías lentas, disonantes y gélidas, indiferentes al caliente cuatro por cuatro del bajo y la batería, logrando un necesario contraste a la monótona letanía del canto rapero. Ser Humano tiene un claro sustrato pop recogido de la calle, limpiado con esmero y devuelto al propio pop. Tiro de Gracia sabe mantener la simplicidad debajo del apretado discurso de las palabras. Sus timbres y melodías tienen delicadeza, sus coros 
están cuidadosamente equilibrados, logrando altos grados de sofisticación en el estribillo, algo digno del mejor pop en español (Juan Pablo González en Revista Rock \& Pop, n. 43, 1997).

El siguiente grupo en ascender a la cúspide fue Makiza. Desde sus filas han surgido nombres destacados del hip-hop chileno: Cristian Bórquez (Seo2), host oficial del torneo de freestyle Red Bull Batalla de los Gallos, y Ana Tijoux, una de las figuras más reconocidas de la música chilena en el contexto internacional. Con la experiencia en el hip-hop adquirida durante los años de exilio político que sufrieron sus respectivos progenitores, los integrantes de Makiza aportaron una perspectiva diferente con respecto a la primera banda examinada, pero igualmente exitosa. En sus letras quedaron retratados temas como la dictadura militar, la transición democrática, las complejidades de insertarse en una sociedad desconocida, las desigualdades que provoca el neoliberalismo, entre otros tópicos de la crítica política y social. Otra novedad introducida fue mezclar los idiomas español y francés al momento de cantar. El primer disco de Makiza había surgido de la autogestión y el segundo contó con una enorme repercusión social, dado que fue producido por Sony Music - Aerolínea Makiza (1999) - .

De esta confluencia igualmente participaron otras bandas, pues el éxito que cosechó Tiro de Gracia como así también Makiza despertó el interés por seguir generando ganancias a partir del fenómeno comercial y mediático que se estaba suscitando. En lo que atañe a la década de 1990, destacan las bandas La Frecuencia Rebelde con su disco homónimo (1999) y Rezonancia con Zorprezaun (1999). Estas agrupaciones grabaron sus respectivos debuts discográficos - únicos álbumes - a través de Sony Music. El alcance de esta multinacional permitió la circulación vigorosa de su música, alcanzando, incluso, un relativo reconocimiento a nivel internacional.

En Chile, el auge del hip-hop de los años noventa también se extendió a aproximadamente los tres primeros años de la década del 2000. En este breve lapso de tiempo Tapia Rabia Jackson grabó discos con EMI (La Conexión, 2000) y Warner Music (Fusión de estilos, 2001; Fusión de estilos V2.0). Por su parte, Némesis -dúo compuesto por algunos ex-integrantes de Makiza - tuvo su debut discográfico con Sony Music (Justicia Divina, 2001). Pese a que estos grupos no alcanzaron los mismos elogios que Tiro de Gracia, Makiza y otras agrupaciones, en virtud de que su reconocimiento se 
limitó a un ámbito local, igualmente se distinguen por su aporte al desarrollo de esta cultura musical en el país.

Mención especial en esta revisión merece la agrupación La Pozze Latina. Su trayectoria da cuenta explícitamente del devenir del hip-hop chileno a partir de su encuentro con majors. Como fue señalado en el apartado anterior, este grupo había sido uno de los pocos que logró grabar música ante la oportunidad que le otorgó Alerce. Para finales de la década obtuvo un contrato con BMG y esa asociación le permitió acceder a mayores recursos de grabación y difusión y, al mismo tiempo, adoptar de lleno aquel sonido pop al que aludía previamente Juan Pablo González. En consecuencia, el disco Detrás del mundo de los espejos (1999) generó un notorio quiebre sonoro con relación a trabajos predecesores de esta banda. El fundador de La Pozze Latina, Jimmy Fernández, explica esta transición estética y de producción musical.

El tipo de BMG me dijo: ¡Qué pasa si ustedes se van a hacer el videoclip a Panamá, y para el disco mejor contratamos a un productor musical! El tipo de BMG sacó un libro con todos los productores que tenía. Comienza a buscar productores musicales, a ver la biografía, los trabajos que ha hecho: Niggaz With Attitude, NWA, el disco Niggaz For Life. Tocó todos los teclados y todos los bajos de ese disco, ¿quién? El señor Colin Wolfe. Ese yo quiero, porque ese disco para mí es un clásico, yo lo escucho hasta el día de hoy. Trajimos a Colin Wolfe a Chile y estuvo 10 días con nosotros. Con él se produjo el disco Desde el mundo de los espejos. Ese es un artista que ha trabajado con Michael Jackson, Madonna, TLC, Dr. Dre, NWA, Snoop Dog, George Clinton, Funkadelie. Ese dato lo podríamos haber encontrado en Alerce, pero de ahí que Alerce suelte el dinero, lo dudo. Este es un tipo que por canción producida estaba pidiendo en aquellos tiempos, te estoy hablando hace 18 años atrás, en aquel tiempo pedía cinco mil a siete mil dólares por canción, y nosotros lo trajimos por 7 mil dólares para el disco entero. Esas son movidas que hace el sello, porque el sello contacta a este productor. [...] Ahí aprendimos mucho nosotros, también en lo que es la producción musical, sobre todo el orden. (Comunicación personal con Jimmy Fernández, 27 de septiembre de 2017).

Este testimonio reafirma la tesis de que una parte de las transformaciones que experimentó el género musical analizado, especialmente en cuanto a sonido, producción y difusión, estuvo determinada por el accionar de las majors. Así también lo valida el productor y beatmaker Gastón Gabarró (2019), quien señala las renovadas condiciones que impusieron sellos como EMI y Sony Music. En su libro autobiográfico Un hijo de la rosa de los vientos, relata la experiencia en el proceso de producción y grabación de los álbumes Ser Humano y Aerolínea Makiza. Señala que la realización de estos discos se caracterizó por la profesionalización en lo que toca al manejo de 
renovada tecnología (uso del teclado secuenciador Kurzweil K2000 y los softwares Cool Edit y Pro Tools). Igualmente apunta un enriquecimiento sonoro a partir de la disposición de instrumentos musicales convencionales. Desde este prisma, quizás el principal aporte de las majors a la trayectoria del hip-hop chileno fue introducir un estándar de hacer y circular la música entre extensas audiencias, una condición que ha tendido a mantenerse con el paso de los años.

Cabe señalar que aquellas bandas que no contaron con la oportunidad de asociarse a un sello multinacional, igualmente observaron materializadas sus respectivas propuestas, aunque desde otras alternativas. En este ámbito es necesario resaltar el rol que desempeñaron las minors. Un caso emblemático se encuentra en Kalimba Records, sello caracterizado por su dedicación exclusiva al rap chileno. De su catálogo emergieron bandas reconocidas - quizás no por extensas audiencias pero sí en la esfera del hip-hop - como fue el caso de Legua York, Calambre, Guerrillero Okulto, Trovadores Tales, entre muchas otras. Por ello, en el instante en que las multinacionales abandonaron el país (años 2000), el impulso del hip-hop en Chile sería sostenido, en parte, por minors especializadas en rap junto con la autogestión, en un panorama de democratización de la producción musical y de la escucha.

Ahora bien, la conjugación entre majors y minors durante el periodo estudiado -junto con otros agentes/factores- gestionó un lugar común de interacciones que le otorgó dinamismo a una actividad musical. En este sentido, Negus (2005) aduce que ante la existencia de una relación dialéctica entre industria y culturas musicales, tanto sellos multinacionales como independientes no pueden ser reducidos a un binarismo antagónico, pues ambas configuraciones se despliegan en un devenir complementario, observable en la continua adaptación e imitación de variadas estrategias de difusión, producción musical e interacción con los artistas ${ }^{5}$. Por tanto, más que exaltar el aporte de uno u otro, lo que sí es innegable, en el caso chileno, es la confluencia que determinó el desarrollo del hip-hop, la cual no habría sido posible sin

\footnotetext{
${ }^{5}$ Canales (2017, p. 30) indica que los músicos de Alerce presionaban a los ejecutivos para que desarrollaran estrategias de difusión, en la medida de lo posible, como aquellas que estaban efectuando las majors.
} 
la red underground de raperos locales, que se gestó desde los primeros años de esta cultura musical en el país.

\section{Algunas transformaciones que experimentó el hip-hop chileno durante el epílogo de los años noventa}

Durante el período estudiado se visualizaron otros cambios en el despliegue del hip-hop en Chile, más allá del hecho de que las majors contribuyeran a delimitar una renovada sonoridad del género - un sonido ecléctico desde el beat y estructuras cuyo eje era el estribillo cantado-, y las estrategias localizadas para generar una difusión más fluida del producto musical -por ejemplo, la grabación de videoclips-

En primer lugar, hay que apuntar la evidente ampliación de la comunidad asociada. Ergo, Salazar y Pinto (2002, p. 275) indican que las culturas musicales (también deportivas) al cabo de la dictadura se masificaron rápidamente entre la juventud, dada la necesidad imperiosa de expresión e identidad. En este ámbito el hiphop había participado a comienzos de la década señalada, aunque sin integrar el grupo de mayor popularidad y relevancia. Pero esta condición cambió a finales de los años noventa, pues se avizoró un aumento ostensible del número de seguidores y de exponentes del rap en Chile. Tal auge podía corroborarse al observar los espacios de reunión en diferentes urbes del país. El caso más representativo corresponde a la capital, Santiago, porque los primeros y emblemáticos sitios de congregación, ubicados en sectores céntricos de la ciudad, debieron abandonarse ante la gran cantidad de personas aglutinadas. Por ello, esta comunidad debió reunirse en territorios amplios (Parque Forestal, Estación Mapocho) ya que estos nuevos lugares contaban con mejores condiciones para una cuantiosa circulación de individuos (RODRÍGUEZ, 2020b). La revista de la época Kultura Hip Hop daba cuenta de esta situación en una de sus editoriales.

Tomando en cuenta la masificación de la música rap tanto en las radios como en T.V., el número de gente que se reunía en Estación Mapocho era inmenso. Tranquilamente podían sobrepasar las 300 personas cada sábado. Durante el 
97 fue el apogeo de Mapocho, muchachos que se sumaban sábado tras sábado siendo el pilar principal ver a los B-Boys practicar. Pero a esto se sumó una disciplina en la que pocos se podían destacar con astucia "Freestyle" o improvisación de rimas, disciplina en la que participaban más muchachos (Kultura Hip Hop, Editorial, vol.3, p. 4, 1998).

Igualmente es ineluctable el auge que estaban adquiriendo otras manifestaciones artísticas integrantes de la cultura hip-hop, tales como el grafiti, los DJ y el break dance. Si bien es cierto que para la época del boom el sitial de privilegio lo ocupaba el rap, de todas maneras el impulso mediático que éste estaba adquiriendo contribuía para generar un auspicioso panorama del hip-hop en sus variadas expresiones y singularidades. En Kultura Hip Hop también aludieron en su momento a dicha circunstancia.

Se debe tomar en cuenta que en los últimos tres años el desarrollo de las disciplinas del Hip-Hop a (sic.) ido en aumento considerablemente, en caso de ver las paredes de nuestra capital y darse cuenta de esto. Los graffytis (sic.) han invadido nuestra ciudad dándole color y vida. Los grupos de Mc's han aumentado enormemente, todos con diferentes estilos y formas de decir las cosas. Los B-Boys se han consolidado y cada vez son más los que quieren desafiar la gravedad, incluso mujeres se están atreviendo, que lo hace más atractivo y llamativo. Los DJ's de música Hip-Hop han ido en aumento, aunque la compra de equipos no está al alcance de todos. Todo lo anterior se deduce que nuestra cultura Hip-Hop va en buen pie (Kultura Hip Hop, Editorial, vol. 3, p.4, 1998).

Otro síntoma del renovado panorama fue que Chile sería considerado como un territorio fructífero para el hip-hop en el contexto internacional. Muestra de aquello fue que el país se convirtió en plaza fija para estrellas mundiales del rap. Así las cosas, grupos como Cypress Hill, Beastie Boys, Public Enemy, Delinquent Habits, entre otros, efectuaron shows multitudinarios en varias oportunidades durante la época convocada. Ante esto, raperos provenientes de otros lugares del subcontinente solían arribar a Chile para presenciar estos conciertos, ya que en sus respectivos países no tenían lugar - un auge inscrito en el próspero contexto de espectáculos de música en vivo que se suscitaron durante la post-dictadura chilena, ante las mayores libertades y reconocimiento social de la diversidad cultural (SAINT-JEAN, 2019) - . Esta cualidad ha trascendido al paso del tiempo y actualmente Chile continúa albergando espectáculos masivos de hip-hop, siendo reconocido como una escena de preeminencia a nivel latinoamericano. 
En síntesis, para finales de los años noventa e inicios de la siguiente década, el hip-hop chileno se había transformado en una cultura musical popular, mediatizada y cercana a las últimas tendencias sonoras globales de este género, en particular. No obstante, su desarrollo no alcanzaría un punto culmen durante esta etapa, ya que en años posteriores el hip-hop ha seguido desenvolviéndose en concordancia tanto con las transformaciones socioculturales del país, como con las continuas mutaciones que ha experimentado la industria discográfica y el campo de la música popular.

\section{Reflexiones finales}

En esta investigación se puso de relieve una operación/etapa poco explorada académicamente. Pese a que la profundización en dichos hechos puede interpretarse como una evocación de aspectos positivos, bajo ningún término se quiso proponer que el "rap noventero" sea el mejor, o que las majors sean entes benefactoras de la música. Por el contrario, reconozco las contradicciones en las que comúnmente incurren estos agentes de la industria cultural cuya exaltación del capital suele depredar las ganancias, generar inseguridad laboral y propiciar desamparo a los grupos. Pero independiente de la perspectiva, sugiero que el estudio de la música debiera siempre contemplar el rol que desempeña "la industria", ya que a partir de su injerencia igualmente se pueden seguir afianzando identidades, discursividades y performances en las diferentes escenas de música popular - lo cual no deja de ser interesante de analizar - .

Siguiendo esta línea, Yúdice (2002, p. 21) resignifica el aporte social de las industrias culturales en América Latina. Más allá de la producción de discos, libros, televisión o radio que generan empleo y dinamizan las economías locales, apunta que estas industrias generan un beneficio a los agentes sociales, ya que las ganancias que se adquieren pueden contribuir para concretar determinadas agendas o visibilizar ciertos discursos de crítica social. Esta posición armoniza parcialmente con el giro epistemológico que ha adquirido la sociología de la música, donde el foco se ha orientado menos hacia el aparato teórico-crítico, y más hacia los sujetos y sus agencias 
para significar la música, aun cuando estos se encuentren insertos en una dinámica capitalista del consumo musical (HERNÁNDEZ SALGAR, 2016).

En efecto, la industria cultural generó una circulación más extendida del rap chileno. Este fue el paso inicial para que, por ejemplo, sujetos excluidos abrazaran el hip-hop. Si bien nos referimos a una época en que el control de la cadena productiva de la música estaba mayormente a cargo de los sellos y los medios de comunicación, no necesariamente estos dictaban, en última instancia, los significados y usos de la música. Así fue como muchos miembros de la comunidad del hip-hop chileno aprovecharon el espacio creado/ampliado/renovado para concretar objetivos individuales, difundir la cultura que los identificaba, vociferar una crítica a la sociedad, u otra acción. Un caso representativo de esta interacción es el de Ana Tijoux (Makiza), quien además de ser una figura mediática que precisamente surgió durante el período, también ha utilizado la música como medio para expresar una diatriba en contra del colonialismo y defensa del medio ambiente (DONOSO, 2018). Su ejemplo ratifica la noción de que no necesariamente se debe estar al margen del mercado para que la música desarrolle una función social.

Firmar por un sello, una multinacional es como una herramienta que nos sirve a nosotros para mostrar más nuestra música, y ahora que los medios se están interesando en el HIP HOP hay que seguir trabajando para que la ilusión crezca aún más. Creo que es súper positivo. Yo encuentro que es increíble que Tiro de Gracia haya vendido 70.000 discos y que se haya generado un mercado nuevo. [...]. Puede que los medios lo saturen, pero la diferencia de fondo que yo veo es que el HIP HOP es una cultura, y encierra toda una filosofía (Entrevista a los integrantes de La Frecuencia Rebelde en revista Kultura Hip Hop, vol. 3, p.30, 1999).

El desdén hacia la industria o nociones de mercado son de larga data en el ámbito de la música. La perspectiva de Theodor W. Adorno otorgó algunas luces, aun cuando se trate de un enfoque bastante refutado en la actualidad. La misma se basa en una crítica a la mercantilización que habitualmente se aplica a la música popular, y cuya manifestación más visible sería la alienación de las audiencias hacia el poder económico. Así, estas formas musicales tendrían un menor rango si se les compara con la llamada música seria, la cual no estaría condicionada por esta clase de fuerzas sociales (ADORNO, 2002). 
Según Szpilbarg y Saferstein (2014, p. 51), quienes analizan los postulados adorniananos junto con los principales aportes teóricos de la Escuela de Frankfurt espacio académico al que estuvo suscrito el pensador alemán-, la denominada teoría crítica estuvo cimentada sobre la expansión e influencia de la industria cultural en las artes. Este marco -aducen- apuntaba a que la forma en que se relaciona el arte con el poder, representado este último en la figura del mercado capitalista y/o industria cultural, determinaba su estimación y correspondiente posición social.

Aun así, la dicotomía entre industria/mercado y arte es un problema sin resolución. Por ejemplo, el sociólogo británico Simon Frith (FRITH apud MEIER, 2008, p. 242) aduce que las valoraciones negativas que suelen hacer las audiencias en música popular corresponden a la medición del capitalismo en cuanto a grado de vinculación con la industria. En un plano académico tal crítica se podría manifestar en la habitual desatención a prácticas musicales muy incorporadas en la industria cultural, ante el recelo de una posible dinámica comercial y, por ende, sumisión a grupos dominantes. Así ha acontecido con el hip-hop chileno ya que, pese a ser ampliamente examinado, sólo se ha estimado su función social de resistencia cultural relacionada con otros períodos de su trayectoria. Su vinculación con las majors, el cine, la televisión o radios masivas, al parecer no condice con la imagen de una cultura musical que se propone otorgar "voz a los sin voz" desde los márgenes del poder.

Por otro lado, la función social de la música en expresiones de índole popular suele rondar nociones políticas y de resistencia (ALABARCES, 2008). Tal clase de uso se exterioriza con mayor vigor en períodos de dictadura o de agitación, y también en contextos con diferencias sociales o cuando el poder económico y político se encuentra concentrado en una minoría (HERRERA, 2011). Sin embargo, postulo que una función social de la música que objetive subvertir discursos o relaciones de poder, no necesariamente deber circunscribirse de manera irremediable a efectuar una censura en letras de canciones o tomar una posición política explícita por parte de los músicos. Para ilustrar esta conjetura me apoyo en dos investigaciones latinoamericanas.

En primer lugar, apunto el examen realizado por Mizrahi (2015) al funk carioca (Brasil). Esta investigadora nos indica que dicha expresión musical ha logrado 
conservar una singularidad definida como anti-hegemónica, no desde una discursividad manifiesta (como habitualmente se realiza en música popular), sino a partir de recursos estéticos, como la apropiación de sonoridades ajenas y manipulación de símbolos. Entonces, la diferencia desde lo estético se manifiesta como el significado subversivo - un postulado que concuerda con lo planteado por Bishnupriya Ghosh acerca de que lo evasivo de la norma desarticula el código semiótico dominante (GHOSH apud BIELETTO, 2019, p. 341) - . Por otro lado, Muñoz (2018) presenta el caso del rap en Buenos Aires, Argentina, indicando que, desde el despertar de los años 2000, la práctica de este género ha estado experimentando un auge, frente a la democratización de tecnologías digitales para la producción musical. Delante de este escenario, y refiriéndose al concepto de desintermediación (GALLO; SEMÁN apud MUÑOZ, 2018), tanto músicos como audiencias del rap bonaerense han adquirido una cierta independencia frente a agentes que históricamente han poseído el control de la música, entre ellos las majors.

Los ejemplos citados tienen atingencia porque dan cuenta de formas alternativas de ejercer la resistencia cultural, lo cual no se sintetiza en una dimensión política, ni tampoco en que las prácticas musicales estén reducidas a determinados usos y significados. En este sentido, Semán (2016) sostiene que los exámenes de prácticas musicales juveniles se han estancado ante una insistencia por develar rasgos hegemónicos y contrahegemónicos y, por lo cual este tipo de prácticas musicales son definidas a partir de una rebeldía inherente. Pero esta categoría resulta problemática al estar interseccionada por variados gustos, usos, valores y significaciones, es decir por el cruce entre lo material y simbólico. De ahí el imperativo de indagar en un todo social (p. 19).

Por tanto, es posible que a partir del estudio de prácticas musicales imbuidas en la industria cultural se puedan descubrir otras funciones sociales de la música, así como diferentes posibilidades de ejercer una resistencia. Pero esto no debiese expresarse en un abandono a la interpelación del mercado o dejar de indagar aspectos hegemónicos y contrahegemónicos de las prácticas musicales. Más bien, los investigadores deberían interrogar al objeto de estudio desde una perspectiva más incluyente y no reducida a ideas prestablecidas $-\mathrm{o}$ respuestas esperadas - . Si 
existiese un valor real de la música, de seguro este no se encuentra ceñido a una sola función social o significación.

\section{Referencias bibliográficas}

ADORNO, Theodor. Sobre la música popular. Guaraguao, Madrid, vol. 6, n.15, p. 163201, 2002.

ALABARCES, Pablo. Posludio: Música popular, identidad, resistencia y tanto ruido (para tan poca furia). Trans. Revista Transcultural de Música, n. 12, [s. n.], 2008.

ALDUNATE, Marcelo. Un testimonio sobre el encuentro de la música chilena y la radio. En: PALOMINOS, Simón (org.). 30 años de la industria musical chilena (1988-2018). Reflexiones y testimonios. Santiago: SCD/Hueders, 2019, p. 110-125.

ARAVENA, Marianela. La escuela de la calle: el hip hop. En: Actas del I Congreso Chileno de Estudios en Música Popular. ¿Qué hay de popular en la música popular?, Santiago: Asociación Chilena de Estudios en Música Popular, 2011, p. 200-209.

ÁVILA, Sofía, LIBERATO, Ana, CONTE, Daniel. O rap em performance resistindo à colonialidade no Brasil: os Brô MC's. Revista Língua E Literatura, Fátima, vol. 19, n. 33, p. 192-207, jun. 2017.

BIELETTO, Natalia. Construcción de la marginalidad de los músicos callejeros. El caso del "Rey Oh Beyve". Revista Cultura y Representaciones Sociales, Ciudad de México, vol. 14, n. 27, p. 309-347, sept. 2019.

CANALES, Jorge. La otra música de los 90'. Alerce: Modernización y marginalización de un sello discográfico independiente. Ámbito Sonoro, Valparaíso, vol. 3, p. 9-35, 2017.

CHANG, Jeff. Generación Hip-Hop. De la guerra de pandillas y el grafiti al gangsta rap. 1. ed. Buenos Aires: Caja Negra, 2015.

DONOSO, Arnaldo. Ecopoesía y descolonización: el rap de Ana Tijoux. Taller de Letras, Santiago, n. 63, p. 11-22, 2018.

EVANS, Claudia, SADE, Bárbara. Lindo momento frente al caos: Músicos Independientes en el Nuevo Chile Global. Tesis para optar al grado de periodista. Escuela de Periodismo, Universidad de Santiago de Chile, Santiago, 2008.

GABARRÓ, Gastón. Un hijo de la rosa de los vientos. 1. ed. Santiago: Autoedición, 2019. 
HERNÁNDEZ SALGAR, Oscar. Los mitos de la música nacional. Poder y emoción en las músicas populares colombianas, 1930-1960.1. ed. Bogotá: Editorial Pontificia Universidad Javeriana, 2016.

HERRERA, Silvia. Un acercamiento al estudio y análisis de la relación música-política. Folios. Publicación de Discusión y Análisis, Jalisco, vol. 4, n. 3, p. 46-53, 2011.

KATZ, Marc. Groove Music. The art and culture of the hip-hop DJ. 1. ed. New York: Oxford University Press, 2012.

MARTIN-CABRERA, Luis. Escribo Rap con R de Revolución: Hip-hop y subjetividades populares en el Chile Actual (2006-2013). Acontracorriente. Una revista de historia social y literatura en América Latina, North Carolina, vol. 14, n. 1, p. 5-36, 2016.

MEIER, Leslie. In excess? Body genres, "bad" music, and the judgment of audiences. Journal of Popular Music Studies, vol. 20, n. 3, p. 240-60, 2008.

MENESES, Lalo. Reyes de la jungla. Historia visual de Panteras Negras. 1. ed. Santiago: Ocho Libros, 2014.

MIZRAHI, Mylene. Englobamiento, subversión y conectividad en el funk carioca. Apuntes CECYP, vol. 17, n. 25, p. 51-91, 2015.

MORAGA, Mario, SOLORZANO, Héctor. Cultura urbana Hip-Hop. Movimiento contracultural emergente en los jóvenes de Iquique. Última Década, Valparaíso, n. 23, p. 77-101, dic. 2005.

MUÑOZ, Sebastián. Entre los nichos y la masividad. El (t)rap de Buenos Aires entre el 2001 y el 2018. Resonancias, vol. 22, n. 43, p. 113-131, jul-nov. 2018.

NEGUS, Keith. Los géneros musicales y la cultura de las multinacionales. 1. ed. Barcelona: Paidós, 2005.

PACINI, Deborah. Oye como va! Hybridity and identity in latino popular music. 1. ed. Filadelfia: Temple University Press, 2009.

POCH, Pedro. Del Mensaje a la Acción Construyendo el Movimiento Hip Hop en Chile (1984-2004 y más allá). 1. ed. Santiago: Quinto Elemento, 2011.

PONCE, David. 30 años de la industria fonográfica en Chile (1988-2018). En: PALOMINOS, Simón (org.). 30 años de la industria musical chilena (1988-2018). Reflexiones y testimonios. 1 ed. Santiago: SCD/Hueders, 2019, p. 30-53.

QUITZOW, Rainer. Lejos de NYC. El hip hop en Chile. Bifurcaciones, Santiago, vol. 2, [s. n.], 2005. 
RODRÍGUEZ, Nelson. Confrontación en la comunidad hip hop chilena por el valor de la autenticidad. Disputa entre la vieja y nueva escuela durante los '90. Tesis para optar al grado de Magíster en Artes mención Musicología. Facultad de Artes, Universidad de Chile, Santiago, 2019.

RODRÍGUEZ, Nelson. El inicio del hip-hop en Chile (1984-1987): La recepción y el aprendizaje de una cultura musical extranjera en tiempos de dictadura. Popular Music Research Today, Salamanca, vol. 2, n. 2, p. 79-99, 2020a.

RODRÍGUEZ, Nelson. El break dance en Santiago de Chile. Auge y declive de un baile que incomoda. En Bieletto, Natalia (org.). Ciudades Vibrantes. Sonido y experiencia aural urbana en América latina. 1 ed. Santiago: Ediciones Universidad Mayor, 2020b.

SAINT JEAN, Alfredo. Conciertos y desconciertos. 30 años de música en vivo en Chile: 1988-2018. En: PALOMINOS Simón (org.). 30 años de industria musical chilena (19882018): Reflexiones y testimonios. Santiago: SCD/Hueders, 2019, p. 96-109.

SALAZAR, Gabriel, PINTO, Julio. Historia Contemporánea de Chile V. Niñez y juventud. 1 ed. Santiago: LOM, 2002.

SEGRETO, Marcelo. A presença da fala na melodia do rap. Música Popular em Revista, Campinas, ano 5, v. 1, p. 7-34, jul.-dic. 2017.

SEMÁN, Pablo. Música, juventud, hegemonía: salidas de la adolescencia. Estudios Sociológicos, vol. 24, n. 100, p. 3-40, 2016.

SZPILBARG, Daniela, SAFERSTEIN, Ezequiel. El concepto de industria cultural como problema: Una mirada desde Adorno, Horkheimer y Benjamin, Bogotá, Calle 14, vol. 9 n. 14, p. 44-57, sept.-dic. 2014.

TIJOUX, Ana María, FACUSE, Marisol, URRUTIA, Miguel. El Hip Hop: ¿Arte popular de lo cotidiano o resistencia táctica a la marginación?. Polis, Revista de la Universidad Bolivariana, Santiago, vol. 11, n. 33, p. 429-449, 2012.

YÚDICE, George. Las industrias culturales: Más allá de la lógica puramente económica, el aporte social. Pensar Iberoamérica, Madrid, vol. 1, n. 1, p. 16-23, 2002.

\section{Grabaciones}

AEROLÍNEA MAKIZA. Makiza. Santiago: Sony Music, 1999.

DETRÁS DEL MUNDO DE LOS ESPEJOS. La Pozze Latina. Santiago: BMG, 1999. 
DECISIÓN. Tiro de Gracia. Santiago: EMI Music, 1998.

FUSIÓN DE ESTILOS. Tapia Rabia Jackson. Santiago: Warner Music, 2001.

FUSIÓN DE ESTILOS V2.0. Tapia Rabia Jackson. Santiago: Warner Music, 2003.

JUSTICIA DIVINA. Némesis. Santiago: Sony Music, 2001.

LA CONEXIÓN. Tapia Rabia Jackson. Santiago: EMI MUSIC, 2000.

LA FRECUENCIA REBELDE. La Frecuencia Rebelde. Santiago: Sony Music, 1999.

RETORNO DE MISERICORDIA. Tiro de Gracia. Santiago: EMI Music, 2001.

SER HUMANO. Tiro de Gracia. Santiago: EMI Music, 1997.

ZORPREZAUN. Rezonancia. Santiago: Sony Music, 1999.

Presentado en: 22/08/2021 Aprobado en: 06/11/2021 Publicado en: 01/12/2021 